\title{
METTL14 suppresses proliferation and metastasis of colorectal cancer by down- regulating oncogenic long non-coding RNA XIST
}

Xiao Yang ${ }^{1,2+}$, Sen Zhang ${ }^{1,2+}$, Changyu He $e^{1 \dagger}$, Pei Xue ${ }^{1,2}$, Luyang Zhang ${ }^{1,2}$, Zirui He ${ }^{1,2}$, Lu Zang ${ }^{1,2}$, Bo Feng ${ }^{1,2^{*}}$, Jing Sun ${ }^{1,2^{*}}$ and Minhua Zheng ${ }^{1,2^{*}}$ (i)

\begin{abstract}
Background: N6-methyladenosine (m6A) is the most prevalent RNA epigenetic regulation in eukaryotic cells. However, understanding of m6A in colorectal cancer (CRC) is very limited. We designed this study to investigate the role of $\mathrm{m} 6 \mathrm{~A}$ in $\mathrm{CRC}$.

Methods: Expression level of METTL14 was extracted from public database and tissue array to investigate the clinical relevance of METTL14 in CRC. Next, gain/loss of function experiment was used to define the role of METTL14 in the progression of CRC. Moreover, transcriptomic sequencing (RNA-seq) was applied to screen the potential targets of METTL14. The specific binding between METTL14 and presumed target was verified by RNA pull-down and RNA immunoprecipitation (RIP) assay. Furthermore, rescue experiment and methylated RNA immunoprecipitation (Me-RIP) were performed to uncover the mechanism.
\end{abstract}

Results: Clinically, loss of METTL14 correlated with unfavorable prognosis of CRC patients. Functionally, knockdown of METTL14 drastically enhanced proliferative and invasive ability of CRC cells in vitro and promoted tumorigenicity and metastasis in vivo. Mechanically, RNA-seq and Me-RIP identified IncRNA XIST as the downstream target of METTL14. Knockdown of METTL14 substantially abolished m6A level of XIST and augmented XIST expression. Moreover, we found that m6A-methylated XIST was recognized by YTHDF2, a m6A reader protein, to mediate the degradation of XIST.

Consistently, XIST expression negatively correlated with METTL14 and YTHDF2 in CRC tissues.

Conclusion: Our findings highlight the function and prognostic value of METTL14 in CRC and extend the understanding of the importance of RNA epigenetics in cancer biology.

Keywords: m6A, METTL14, Long non-coding RNA, Colorectal cancer, RNA epigenetics

\section{Background}

Colorectal cancer (CRC) continues to be a severe health problem worldwide, resulting in 700,000 deaths annually [1]. The mortality of CRC is primarily due to postoperational recurrence and metastasis [2]. Despite of recent advances in therapeutic strategies, including modified

\footnotetext{
*Correspondence: fengborih@126.com; sunjing19820616@163.com; Zhengmhrihospital@163.com

${ }^{\dagger}$ Xiao Yang, Sen Zhang and Changyu He contributed equally to this work. ${ }^{1}$ Division of Gastrointestinal and Colorectal Surgery, Ruijin Hospital, Department of General Surgery, Shanghai Jiao Tong University, School of Medicine, Shanghai 200001, China

Full list of author information is available at the end of the article
}

surgical techniques and improved adjuvant therapy, the prognosis of CRC is still far from satisfactory [3]. Thus, understanding the molecular mechanisms of CRC carcinogenesis and progression is essential to future diagnostic and therapeutic inventions.

Traditionally, epigenetic regulation refers to chemical modifications on DNA or histones, which regulates gene expression independent of genome sequences alterations [4]. Dysregulation of epigenetic modifying enzymes profoundly contributes to human diseases and has been frequently reported in multiple types of cancer [5]. Similarly, RNAs also carry hundreds of various sites for distinct post- 
transcriptional modifications. N6-methyladenosine (m6A) is the most predominant modification of mRNA in eukaryotic cells $[6,7]$. It is a reversible chemical process dynamically controlled by the balanced activities of m6A methyltransferases and demethylases. Since m6A modification has been demonstrated to play a vital role in RNA translation, stability and alternative splicing, perturbations of m6A components are associated with human diseases, especially with cancers [8-10]. Dysregulation of m6A "writer" protein, methyltransferase-like 3 (METTL3) and methyltransferaselike 14 (METTL14), has been reported in liver cancer [11, 12], lung cancer [9] and glioblastoma [13]. Besides, the m6A "eraser", fat mass- and obesity-associated protein (FTO), was found to act as oncogene in acute myeloid leukemia [14] and lung squamous cell carcinoma [15]. However, the status of m6A and the underlying mechanisms in CRC still remain largely unknown.

$\mathrm{X}$ inactivate-specific transcript (XIST) is a newly identified lncRNA which serves as an oncogene in several types of solid tumors including lung cancer [16], ovarian cancer [17], liver cancer [18], and colorectal cancer [19]. The molecular mechanism governing the action of this lncRNA involves its activating effects on a variety of tumorigenic signal pathways. For instance, XIST was shown to expedite proliferation and metastasis of CRC via sequestering miR200b and promoting epithelial-mesenchymal transition [19]. Importantly, empowered by individual-nucleotide resolution UV crosslinking and immunoprecipitation (iCLIP) method, recent m6A mapping study has identified at least $78 \mathrm{~m} 6 \mathrm{~A}$ residues distributed along XIST [20]. Moreover, m6A methylation on special sites is indispensable for XIST to induce transcriptional silencing of genes on the $\mathrm{X}$ chromosome [20]. These results imply that m6A may play a crucial role in the regulation of lncRNAs, which reminds us to explore its potential effects on human cancers, especially on CRC.

In this study, we investigated the role of METTL14, a major m6A "writer", in CRC and addressed the underlying mechanism. We found that METTL14 suppressed proliferation and invasion of CRC cells by down-regulating oncogenic lncRNA XIST in a m6A dependent manner. In line with this, knockdown of METTL14 in CRC cells resulted in decreased m6A-methylation levels of XIST, leading to elevated XIST levels and enhanced tumor driving effects. In total, we highlight the important role of METTL14 and m6A in colorectal cancer and provide a promising marker for predicting prognosis of CRC.

\section{Methods}

\section{Patients and samples}

Tumor tissues and adjacent normal tissues $(>5 \mathrm{~cm}$ away from tumor) were collected from $37 \mathrm{CRC}$ patients undergoing radical surgery in our institute. Clinical parameters including age, sex, stage, pathological diagnosis, TNM status and recurrence were also collected. No patient had received local or systemic treatment before operation. Fresh tumor and paired normal tissues were frozen in liquid nitrogen and stored at $-80^{\circ} \mathrm{C}$. The rest part of specimens was fixed in the formalin and embedded in paraffin for pathological and immunohistochemical (IHC) analysis. Our study protocol has been approved by the Ethics Committee of Ruijin Hospital, Shanghai Jiao Tong University, School of Medicine. Informed consent was obtained from each enrolled patient.

\section{IHC analysis}

Tissue sections were deparaffinized, rehydrated, and microwaved-heated in sodium citrate buffer $(10 \mathrm{mmol} / \mathrm{L}$, $\mathrm{pH}$ 6.0) for antigen retrieval. Then, the slides were incubated with primary antibody. Then the expression levels of target proteins in tissue were examined by two independent pathologists blinded to the clinical characteristics of the patients according to proportion of cell staining $(0=0 \%, 1=\leq$ $25 \%, 2=26$ to $50 \%, 3=51$ to $75 \%, 4=>75 \%$ positive cells) and the staining intensity $(0=$ no staining, $1=$ weak, $2=$ moderate, $3=$ strong). A final score was calculated by multiplying the above two scores. Protein expression was considered high if the final score was greater than 6 points and low if the final score was 6 points or less.

\section{Cell culture and transfection}

Human colon epithelial cell line NCM460 was purchased from the Cell Bank of Type Culture Collection of the Chinese Academy of Sciences (Shanghai, China). Five CRC cell lines (SW480, SW620, HCT116, LoVo, and HT29) were previously purchased from the American Type Culture Collection (ATCC, Manassas, VA, USA) and preserved in the research institute of general surgery. SW480 and NCM460 cells were cultured in RPMI-1640 medium. HT29 and HCT116 were cultured in McCoy's 5A medium, SW620 cells were cultured in Leibovitz's L-15 medium. LoVo was cultured in Ham's F-12 K (Kaighn's) Medium. All kinds of medium were purchased from Invitrogen (Carlsbad, CA, USA) and supplemented with $10 \%$ fetal bovine serum (FBS, HyClone, Logan, USA), $100 \mathrm{U} / \mathrm{ml}$ penicillin and $100 \mu \mathrm{g} / \mathrm{ml}$ streptomycin. Cells were maintained in a $37{ }^{\circ} \mathrm{C}$ incubator with $5 \% \mathrm{CO}_{2}$. The full length METTL14 cDNA was synthesized by RT-PCR from normal colon epithelial cells (NCM460) and then sub-cloned into the pcDNA3.1 vector to construct pcDNA-METTL14 overexpression (METTL14-OE) plasmid. The shRNA sequences targeting METTL14 were synthesized with a wellestablished annealing method and then cloned into pLKO.1 plasmid (Sigma Aldrich, St. Louis, USA). SiRNAs (siMETTL14, siXIST, siWTAP) and negative control were purchased from HanYin Bio-Tech Co.,Ltd. (Shanghai, China). For transient transfection, cells were transfected with plasmids encoding target sequences or siRNAs using 
Lipofectamine 3000 reagent (Invitrogen) according to the manufacturer's instructions. Stable clones were selected by puromycin $(1 \mu \mathrm{g} / \mathrm{ml})$. All transfects were tested regularly by western blot to ensure the efficiency of over-expression or knockdown. Details of sequences or hairpin sequences used in this study were listed in Additional file 2: Table S2.

\section{Cell proliferation and invasion assay}

Cell viability was measured with cell count assay and colony formation assay. For cell count assay, control and transfected cells were cultured in a 96-well plate (3000 cells/well). Triplicate wells were measured in each group. Cell viability was determined every $24 \mathrm{~h}$. The plate was incubated at $37^{\circ} \mathrm{C}$ for $2 \mathrm{~h}$ after each well was added with $10 \mu$ l CCK-8 solution. Then the spectrophotometric absorbance was measured at $450 \mathrm{~nm}$ for each sample. For the colony formation assay, a certain number of control or transfected cells were planted into a six-well plate and maintained in culture media containing 10\% FBS for 2 weeks. Colonies were fixed with methanol and stained with crystal violet (Sigma-Aldrich). The colony number was determined by counting stained colonies using ImageJ software. Transwell invasion assay were performed as we described elsewhere. Briefly, $8 \times 10^{4}$ cells in $200 \mu \mathrm{L}$ serum-free media were seeded in the top chamber (8.0 $\mu \mathrm{m}$ pore size, Corning, USA) with membrane coated by Matrigel (BD Bioscience, USA). $600 \mu \mathrm{L}$ of $10 \%$ FBScontaining medium was placed into the bottom chamber as an attractant. After incubation for $24 \mathrm{~h}$, the cells that did not invade to the lower side of the chamber were removed from the top side. The chambers were then stained with crystal violet and photographed.

\section{Western blot}

Briefly, cells were washed with PBS and lysed with RIPA buffer (Thermo Scientific, MA, USA). The lysates were then sonicated on ice and centrifuged at $14,000 \mathrm{~g}$ for 5 min at $4{ }^{\circ} \mathrm{C}$ to remove cell debris. Next, the supernatants were resolved in $12.5 \%$ SDS-PAGE (30 $\mu \mathrm{g} /$ lane) and transferred onto 0.22-lm polyvinylidene fluoride (PVDF) membranes (Millipore, MA, USA). The membranes were probed with proper antibodies overnight at $4{ }^{\circ} \mathrm{C}$. After washing for three times, the membrane was incubated in HRP-labeled secondary antibody for $2 \mathrm{~h}$ at room temperature. Protein bands were revealed by enhanced chemiluminescence (ECL) method. The following antibodies used in this study were purchased from Cell Signaling Technology (Beverly, MA, USA): rabbit anti-METTL14 (D8K8W), rabbit anti-WTAP (56501), rabbit anti-GAPDH (D6H11).

\section{RNA extraction and real-time PCR}

Total RNA was extracted using TRIzol reagent (Ambion) and cDNA synthesis was performed using a reverse transcription kit (Promega, Madison, USA) according to the manufacturer's instructions. PCR was conducted using the SYBR Green Master Mix (Applied Biosystems, MA, USA) and the Applied Biosystems 7900HT sequence detection system. After the reactions were complete, relative gene expression level was calculated using the $2^{-\Delta \Delta C t}$ method. GAPDH was used as an endogenous control. Primers used were as follows: METTL14: sense: 5'-GAACACAGAGCTTAAATCCCCA-3'; antisense: 5'-TGTCA GCTAAACCTACATCCCTG-3'. XIST: sense: 5'-GCATA ACTCGGCTTAGGGCT-3', antisense: $5^{\prime}$-'TCCTCTGCCT GACCTGCTAT-3'.

\section{RNA immunoprecipitation}

Cells cultured in $10 \mathrm{~cm}$ plate was washed twice with icecold PBS and scraped off in $1 \mathrm{~mL}$ PBS. Then the cell was centrifuged and re-suspended in an equal pellet volume of complete RIP lysis buffer (Merck Millipore). $5 \mu \mathrm{g}$ antibody was pre-bound to Protein A/G magnetic beads in immunoprecipitation buffer $(20 \mathrm{mM}$ Tris- $\mathrm{HCl} \mathrm{pH} 7.5,140 \mathrm{mM}$ $\mathrm{NaCl}, 0.05 \%$ TritonX-100) for $2 \mathrm{~h}$ and then incubated with $100 \mu \mathrm{l}$ cell lysates over night at $4{ }^{\circ} \mathrm{C}$ with rotation. Then RNA was eluted from the beads by incubating with $400 \mu \mathrm{l}$ elution buffer for $2 \mathrm{~h}$. The eluted RNA was precipitated with ethanol and dissolved with RNase-free water. Enrichment of certain fragments was determined by real-time PCR. Primer used for XIST quantification was designed as follows: sense: 5'-CTGCTGCAGCCATATTTCTTAC-3', anti-sense: 5'-TACGCCATA AAGGGTGTTGG-3'. IgG was used for negative control. For m6A-RNA immunoprecipitation (Me-RIP), XIST extracted from equal amount cell lysates was used as input to measure the m6A-methylated rate of XIST. Antibodies used in this experiment were as follows: anti-m6A (ab190886, Abcam), anti-YTHDF1 (ab99 080, Abcam), anti-YTHDF2 (ab170118, Abcam), anti-YTH DF3 (ab103328, Abcam), anti-YTHDC1 (ab122340, Abca m), anti-YTHDC2 (ab176846, Abcam). Anti-IgG (Cell Signaling Technology, \#2729).

\section{RNA-binding protein pull-down assay}

RNA pull-down assay was performed with RNA-Protein Pull-Down Kit (Pierce, USA) according to the protocol of manufacturer. Briefly, full length of XIST was transcribed in vitro using Large Scale RNA Production Systems (Promega, USA) and labeled with Biotin using Biotin RNA Labeling Mix (Roche, Switzerland). Then 1 mg cell lysates extracted from CRC cells was incubated with $3 \mu \mathrm{g}$ purified biotinylated transcripts for $1 \mathrm{~h}$ at $4{ }^{\circ} \mathrm{C}$ with rotation. Then the streptavidin agarose beads were added into cell protein lysate to precipitate the RNAprotein complex. The beads were washed three times and boiled in sodium dodecyl sulfate (SDS) buffer to retrieve proteins for western blot analysis. 


\section{RNA-seq and analyses}

For RNA sequencing, purified RNA from shMETTL14 and control cells was used for library construction with Illumina TruSeq RNA Sample Prep Kit (FC-122-1001) and then sequenced with Illumina HiSeq 2000. Raw reads were aligned to the human genome GRCh37/hg19 by Bowtie2. Differentially expressed genes (DEGs) between treatment and control samples were identified with limma-voom method. A heatmap clustered by k-means was used to show DEGs or transcripts.

\section{Animal experiments}

Establishment and analysis of nude mice (male BALB/c $\mathrm{nu} / \mathrm{nu}$ nude mice, 4-week-old) subcutaneous xenograft model was performed as we described before [21]. For liver metastasis model, mice were anaesthetized and an incision was made through the skin and peritoneum to expose the spleen. $1 \times 10^{6}$ HCT116 cells were injected into the spleen ( $n=4$ each group). All mice were kept until death due to the neoplastic progression or until the end of the experiment ( 6 weeks). The mice livers were removed and carefully dissected to evaluate the metastatic lesions. Animal experiments were all carried out according to the Guide for the Care and Use Laboratory Animals of Ruijin Hospital, Shanghai Jiao Tong University School of Medicine.

\section{Statistical analysis}

$\mathrm{R}$ software (version 3.5.1) was used for statistical analysis and data visualization. Protein and RNA levels were compared using two-tailed Student's $t$ test. Correlations between METTL14 expression in CRC tissues and clinicopathological features were analyzed with Pearson Chisquare $(\mathrm{X} 2)$ test. Overall survival (OS) and recurrence free survival (RFS) was assessed by Kaplan-Meier method, difference between survival curves was determined by logrank test. Differences with a $p$ value $<0.05$ were considered as statistically significant.

\section{Results}

\section{METTL14 was downregulated in CRC}

To investigate the potential role of $\mathrm{m} 6 \mathrm{~A}$ in colorectal cancer, we first detected the mRNA levels of major m6A methyltransferase including METTL3 and METTL14 in 37 CRC and paired normal samples. As shown, METTL14 was remarkably down-regulated in cancerous compared to paired normal samples (Fig. 1a, left panel). In contrast, no significant difference was observed in the expression level of METTL3 (Fig. 1a, right panel). Moreover, similar expression pattern of METTL14 and METTL3 (Fig. 1b) in CRC were validated in an expanded cohort containing 387 cases of CRC patients from Gene Express Omnibus (GEO) dataset (GSE14333), which further supported our initial findings. However, analysis of RNA sequencing data from The Cancer Genome Atlas (TCGA) shown decreased
METTL14 (Additional file 3: Figure S1A) but elevated METTL3 levels (Additional file 3: Figure S1B) in human $\mathrm{CRC}$ tissue relative to normal tissue. Due to the inconsistency of METTL3 analysis results, we chose METTL14 for further research in this study. Tissue microarray containing all 37 pairs of cancerous and matched normal tissue was analyzed by immunohistochemical (IHC) staining (Fig. 1c). Consistently, high METTL14 expression was observed in $67.6 \%(25 / 37)$ of normal tissues. Meanwhile, in paired CRC tissue, only $32.4 \%(12 / 37)$ cases showed high METTL14 signal $(p=0.002)$. Besides, loss of METTL14 was also observed in multiple human CRC cell lines (Fig. 1d). With above evidence, we concluded that METTL14 was frequently down-regulated in human CRC and might be implicated in pathogenesis and progression of CRC.

\section{Loss of METTL14 predicted unfavorable prognosis of CRC patients}

Next, we sought to explore the clinical significance of METTL14 by performing correlation analysis of METTL14 levels with clinicopathological features of enrolled CRC patients. As shown, low expression of METTL14 was positively correlated with larger tumor size, lymphatic invasion, remote metastasis and more advanced TNM stage (Table 1), indicating METTL14 might play an important role in modulating proliferation and invasion of CRC. Moreover, Kaplan-Meier analysis revealed that patients with low METTL14 expression exhibited unfavorable recurrence free survival (RFS, Fig. 1e). Moreover, we established an independent validation cohort by extracting RNA-sequencing and clinical data of CRC patients from GSE14333 dataset. Consistent with our results, decreased METTL14 expression also implicated worse RFS (Fig. 1f). In addition, multivariate analysis showed that METTL14 was an independent risk factor for RFS of CRC patients from our cohort (Table.2) and GEO dataset (Fig. 1g). Time-dependent ROC analysis showed that METTL14 had stronger predictive ability than TNM stage, especially in predicting 24-month RFS (Fig. 1h, Additional file 4: Figure S2A). Furthermore, TCGA data also showed that METTL14 was positively correlated with OS (Additional file 4: Figure S2B) and represented as an independent risk factor (Additional file 4: Figure S2C). These results suggested METTL14 is a reliable prognostic marker of CRC patients.

\section{Knockdown of METTL14 promoted proliferation and invasion of CRC}

Given the fact that METTL14 is down-regulated in tumor tissue, we speculated that METTL14 may act as a tumor suppressor in CRC. To define the functional roles of METTL14 in CRC, we established METTL14-knockdown cell model in HCT116 and HT29 cells with two independent siRNAs (Fig. 2a, Additional file 4: Figure S2D). The alteration of proliferative ability of CRC cells was then 


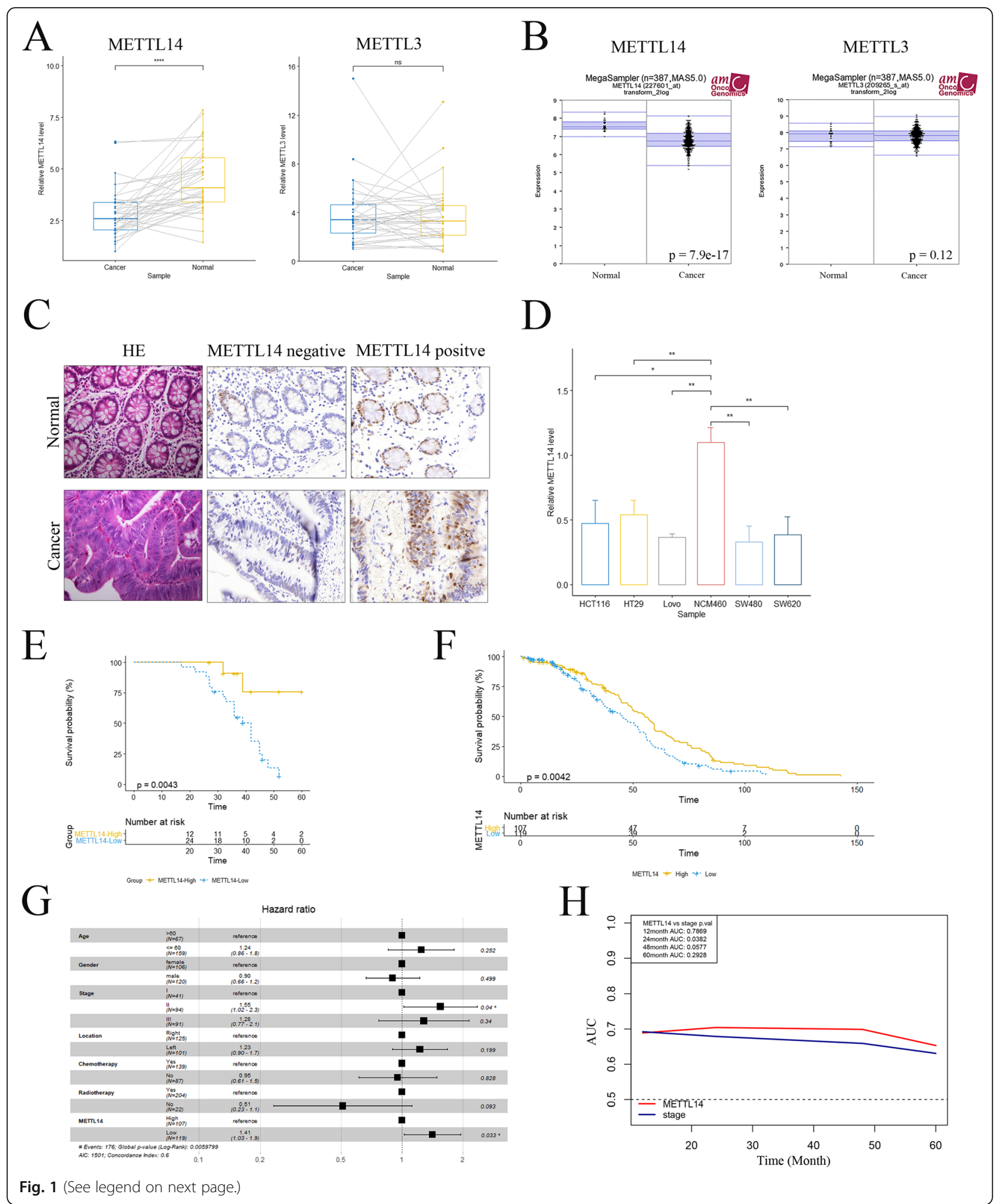


(See figure on previous page.)

Fig. 1 METTL14 was down-regulated in human colorectal cancer. a Real-time PCR analysis of METTL14 and METTL3 expression levels in colorectal cancer and paired normal tissue. ns, not significant. ${ }^{* *}, p<0.01$. b Mega-sampler analysis of expression patterns of METTL14 and METTL3 in cancer and normal tissues from GEO dataset (GSE14333). $P$ values were as indicated. $\mathbf{c}$ Representative images of immunohistochemical (IHC) staining of METTL14 in colorectal cancer and paired normal tissue. $\mathbf{d}$ Expression levels of METTL14 in different CRC cell lines and normal NCM460 cells. ${ }^{* *}, p<0.01$. e-f Kaplan-Meier analysis of RFS of CRC patients from our cohort (e) and GSE14333 dataset (f). $\mathbf{g}$ Multivariate analysis of clinical prognostic parameters for RFS of CRC patients in GSE14333 cohort. P values were as indicated. $\mathbf{h}$ Time-dependent ROC analysis showing the predictive ability of METTL14 of RFS of CRC patients. P values were as indicated. PCR was analyzed by paired student's t-test (a) and nonpaired student's t-test (d). Mega-sampler analysis of GEO data (b) was generated by R2 online tools (http://r2.amc.n//). Difference of RFS (e and $\mathbf{f}$ ) between two subgroups was analyzed with Log-rank test. Time-dependent ROC analysis was constructed with Cox model from 'Survival' R package

evaluated by cell count assay and colony formation assay, respectively. As expected, depletion of METTL14 markedly enhanced proliferative ability of CRC cells, reflected by expediated growth rate (Fig. 2b) and increase of colony number (Fig. 2c) of siMETTL14 cells. In addition, transwell invasion assay showed that knockdown of METTL14 dramatically promoted invasive ability of CRC cells (Fig.

Table 1 Relationship between METTL14 and clinicopathologic factors of CRC patients

\begin{tabular}{|c|c|c|c|c|}
\hline \multirow[t]{2}{*}{ Variables } & \multirow{2}{*}{$\begin{array}{l}\text { Case } \\
(N=37)\end{array}$} & \multicolumn{2}{|c|}{ METTL14 level } & \multirow[t]{2}{*}{$p$ value } \\
\hline & & $\begin{array}{l}\operatorname{High}(n= \\
12)\end{array}$ & $\begin{array}{l}\operatorname{low}(n= \\
25)\end{array}$ & \\
\hline Gender & & & & 0.85 \\
\hline Male & 27 & 9 & 18 & \\
\hline Female & 10 & 3 & 7 & \\
\hline Age & & & & 0.40 \\
\hline$\leq 60$ & 16 & 4 & 12 & \\
\hline$>60$ & 21 & 8 & 13 & \\
\hline Location & & & & 0.638 \\
\hline Ascending colon & 9 & 3 & 6 & \\
\hline Transverse colon & 11 & 2 & 9 & \\
\hline Descending colon & 2 & 1 & 1 & \\
\hline $\begin{array}{l}\text { Sigmoid colon and } \\
\text { Rectum }\end{array}$ & 15 & 6 & 9 & \\
\hline Tumor size & & & & 0.003 \\
\hline$\leq 5 \mathrm{~cm}$ & 15 & 9 & 6 & \\
\hline$>5 \mathrm{~cm}$ & 22 & 3 & 19 & \\
\hline Local invasion & & & & 0.127 \\
\hline $\mathrm{T} 1+\mathrm{T} 2$ & 15 & 7 & 8 & \\
\hline $\mathrm{T} 3+\mathrm{T} 4$ & 22 & 5 & 17 & \\
\hline Lymphatic invasion & & & & 0.001 \\
\hline No & 10 & 8 & 2 & \\
\hline $\mathrm{N} 1+\mathrm{N} 2$ & 27 & 4 & 23 & \\
\hline Remote metastasis & & & & 0.042 \\
\hline MO & 30 & 12 & 18 & \\
\hline M1 & 7 & 0 & 7 & \\
\hline TNM stage & & & & 0.001 \\
\hline$|+1|$ & 10 & 8 & 2 & \\
\hline$\| I I+I V$ & 27 & 4 & 23 & \\
\hline
\end{tabular}

2d). Moreover, two shRNAs targeting METTL14 (Additional file 4: Figure S2E) were designed and transfected into CRC cell lines to further validate the alterations of phenotypes of CRC cells. Consistently with above results, shMETTL14 cells demonstrated enhanced growing (Additional file 5: Figure S3A, S3B) and invasive (Additional file 5: Figure S3C) ability compared to control cells in cell count assay, colony formation assay and transwell invasion assay. To test our in vitro findings, we established shMETTL14 cell models and injected HCT116shMETTL14 and control cells subcutaneously in the flank of nude mice to evaluate the effect of METTL14 on CRC tumorigenicity (Fig. 3a). Consistent with our in vitro observations, knockdown of METTL14 resulted in significant increase of the tumor volume (Fig. $3 \mathrm{~b}$ ) and tumor weight (Fig. 3c). Besides, significant higher ki-67 rate was observed in the xenograft tumors formed by shMETTL14 cells by IHC staining (Fig. 3d and e). Furthermore, we generated a liver metastasis model by injecting tumor cells into the spleen of nude mice. As expected, more metastatic nodules were found in shMETTL14 group compared to their control counterparts (Fig. 3f). Collectively, these results suggested knockdown of METTL14 promoted proliferation and invasion of CRC in vitro and in vivo.

\section{METTL14 inhibited CRC growth and metastasis by targeting IncRNA XIST}

We next sought to investigate the mechanism by which METTL14 suppressed malignant phenotype of CRC cells. As a key m6A methyltransferase, METTL14 induces installation of methyl group on m6A residues of target RNAs and activates downstream signaling. Moreover, recent studies demonstrated that pri-miRNAs could be marked by m6A-methylation for subsequent splicing and maturing [22]. Besides, m6A process was also proved to participate in modulating process of IncRNAs [23]. These reports not only highlight the important role of m6A modification in RNA processing, but also remind us that m6A-methylation can affect cancer biology by regulating non-coding RNAs (ncRNAs). We then conducted gene set enrichment analysis (GSEA) to explore the potential downstream pathways of METTL14. As shown, METTL14 was positively correlated with ncRNA metabolism (Additional file 6: Figure S4A), ncRNA processing (Additional file 6: Figure S4B) and 
Table 2 Univariate and multivariate analysis of clinicopathologic factors for RFS of CRC patients in our cohort

\begin{tabular}{|c|c|c|c|c|c|c|}
\hline \multirow[b]{2}{*}{ Variables } & \multicolumn{3}{|c|}{ Univariate analysis } & \multicolumn{3}{|c|}{ Multivariate analysis } \\
\hline & Hazard ratio & $P$ value & $95 \% \mathrm{Cl}$ & $\mathrm{HR}$ & $P$ value & $95 \% \mathrm{Cl}$ \\
\hline Age $(\leq 60$ vs $>60)$ & 1.71 & 0.309 & $0.61-4.76$ & & & \\
\hline Gender & 0.875 & 0.760 & $0.37-2.06$ & & & \\
\hline \multicolumn{7}{|l|}{ Location } \\
\hline Ascending colon & 1.40 & 0.574 & $0.43-4.48$ & & & \\
\hline Transverse colon & 2.38 & 0.156 & $0.72-7.90$ & & & \\
\hline Descending colon & 0.82 & 0.856 & $0.90-7.10$ & & & \\
\hline Sigmoid colon and Rectum & 1.00 & 0.490 & .. & & & \\
\hline Tumor size $(\leq 5 \mathrm{~cm}$ vs $>5 \mathrm{~cm})$ & 2.33 & 0.128 & $0.78-6.91$ & & & \\
\hline Stage $(I+\|$ vs ||$I+I V)$ & 2.16 & 0.176 & $0.71-6.55$ & & & \\
\hline Local invasion (T1 + T2 vs T3 + T4) & 0.525 & 0.140 & $0.22-1.23$ & & & \\
\hline Lymphatic invasion (N0 vs N1 + N2) & 2.16 & 0.176 & $0.71-6.55$ & & & \\
\hline Remote metastasis ( $\mathrm{M} 0 \mathrm{vs} \mathrm{M} 1$ ) & 6.35 & 0.001 & $2.13-18.91$ & 7.02 & 0.008 & $1.65-29.87$ \\
\hline METTL14 level (low vs high) & 6.26 & 0.014 & $1.44-27.18$ & 21.02 & 0.011 & $2.04-216.78$ \\
\hline
\end{tabular}

RNA degradation (Additional file 6: Figure S4C). In contrast, negative correlation was seen between METTL14 and several signaling pathways namely growth factor binding, collagen trimer and collagen binding (Additional file 6: Figure S4D-4F). Therefore, METTL14 may exert anti-tumor activity via targeting and down-regulating oncogenes or oncogenic lncRNAs in these pathways. We then performed RNA-seq to interrogate the expression changes in HCT116-shMETTL14 cells relative to the control cells. The fold change of gene expression was calculated and genes with $\log 2|\mathrm{FC}|>0.5$ were considered as differentially expressed. Among thousands of potential targets, we noticed lncRNA XIST was significantly upregulated upon METTL14 knockdown (Fig. 4a, Additional file 7: Figure S5A). XIST is a potent oncogenic lncRNA expediting growth and metastasis of CRC cells [19]. Importantly, analysis of transcriptome m6A mapping data revealed that at least $78 \mathrm{~m} 6 \mathrm{~A}$ residues are located across XIST sequence (Additional file 7: Figure S5B and Additional file 1: Table S1) [20]. As typical mRNA only contains $3-5$ m6A sites, the high abundance of m6A sites of XIST implied an incredibly important role of $\mathrm{m} 6 \mathrm{~A}$ in regulating of XIST function. Thus, we assumed that METTL14 inhibited CRC growth and metastasis by down-regulating lncRNA XIST. To verify this hypothesis, expression levels of XIST was first detected in METTL14 knockdown and control cells. As expected, METTL14 depletion led to distinct elevation of XIST in two CRC cell lines (Fig. 4b). Next, we quantified XIST levels in cancer and normal specimens. Consistent with reported results, XIST exhibited higher levels in cancer compared to normal tissues (Fig. 4c), which suggested a tumor-driving effect of XIST in CRC. Moreover, a negative correlation was observed between expression levels of METTL14 and XIST in CRC samples from our cohort (Fig. 4d). Besides, TCGA RNA-seq data of CRC also showed that XIST upregulation often happened in cases with downregulation of METTL14 (Fig. 4e). Additionally, enhanced cell growth (Fig. 4f, g and Additional file 7: Figure S5C) and invasion (Additional file 7: Figure S5D) induced by METTL14 knockdown could be attenuated by XIST inhibition. Notably, these results were also tested in shMETTL14 cells to confirm that knockdown of XIST diminished the growth (Additional file 8: Figure S6A, S6B) and invasion (Additional file 8: Figure S6C) of shMETTL14 cells. Taken together, these results indicated that increased CRC growth and invasion resulted from METTL14 depletion was, at least partly, due to elevation of lncRNA XIST.

\section{METTL14 down-regulated XIST through a m6A-YTHDF2 dependent pathway}

We next plan to clarify whether the function of METTL14 down-regulating XIST directly depends on its m6A catalytic activity. METTL14 was ectopically over-expressed in HCT116 and HT29 cells (Fig. 5a, Additional file 9: Figure S7A), the potential alterations of XIST expression level and cell phenotypes were then assessed. As shown, overexpression of METTL14 resulted in remarkable decrease in XIST expression level (Fig. 5b), cell growth (Fig. 5c, d) and invasion (Fig. 5e and Additional file 9: Figure S7B) of CRC cells, which were consistent with the above results and confirmed the suppressive effects of METTL14 on CRC. Recently, METTL3 and METTL14 are identified as interactors with Wilms tumor-associated protein (WTAP). WTAP acts as a scaffold and binds the methyltransferase to form a complex which mediates m6A methylation on RNAs. The loss of WTAP significantly blocks m6A modification process. Thus, we performed WTAP knockdown with siRNA in METTL14-overexpressed (METTL14-OE) cells (Fig. 5a, 


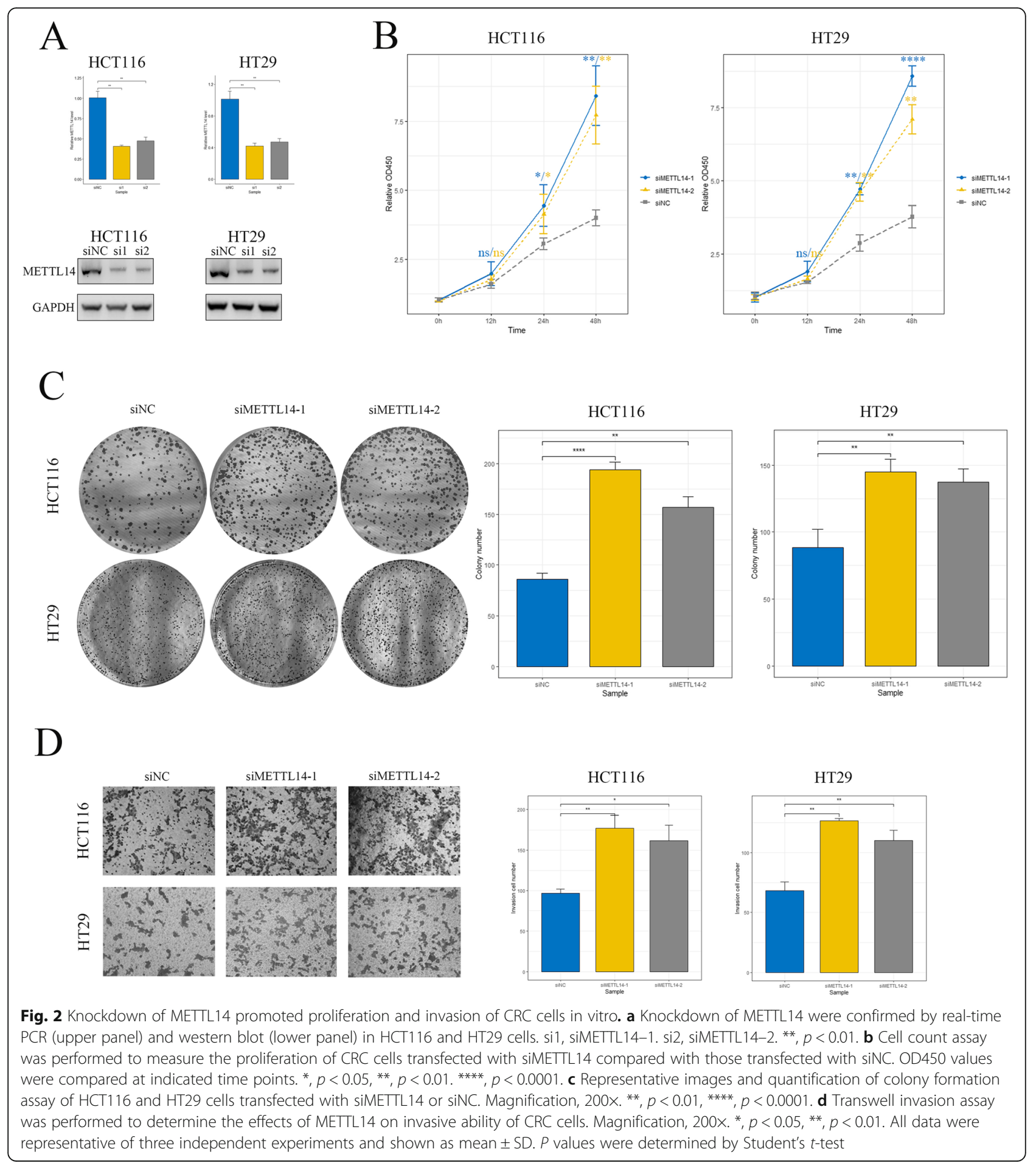

Additional file 9: Figure S7A) to inhibited the cellular m6A process and analyzed the effect of this act. As shown, the decreased XIST level induced by METTL14 overexpression could be attenuated by WTAP depletion (Fig. 5b). In line with this, WTAP knockdown significantly rescued the impaired growth (Fig. 5c and d) and invasion (Fig. 5e and Additional file 9: Figure S7B) of METTL14-OE cells. These results suggested that the downregulation of XIST induced by METTL14 was dependent on the formation of m6A complex and subsequent m6A methylation process. Moreover, m6A-methylated XIST was immunoprecipitated with m6A antibody in siMETTL14 and siWTAP cell lysates and then quantified with real-time PCR. We found that knockdown of METTL14, or WTAP led to significant reduced 


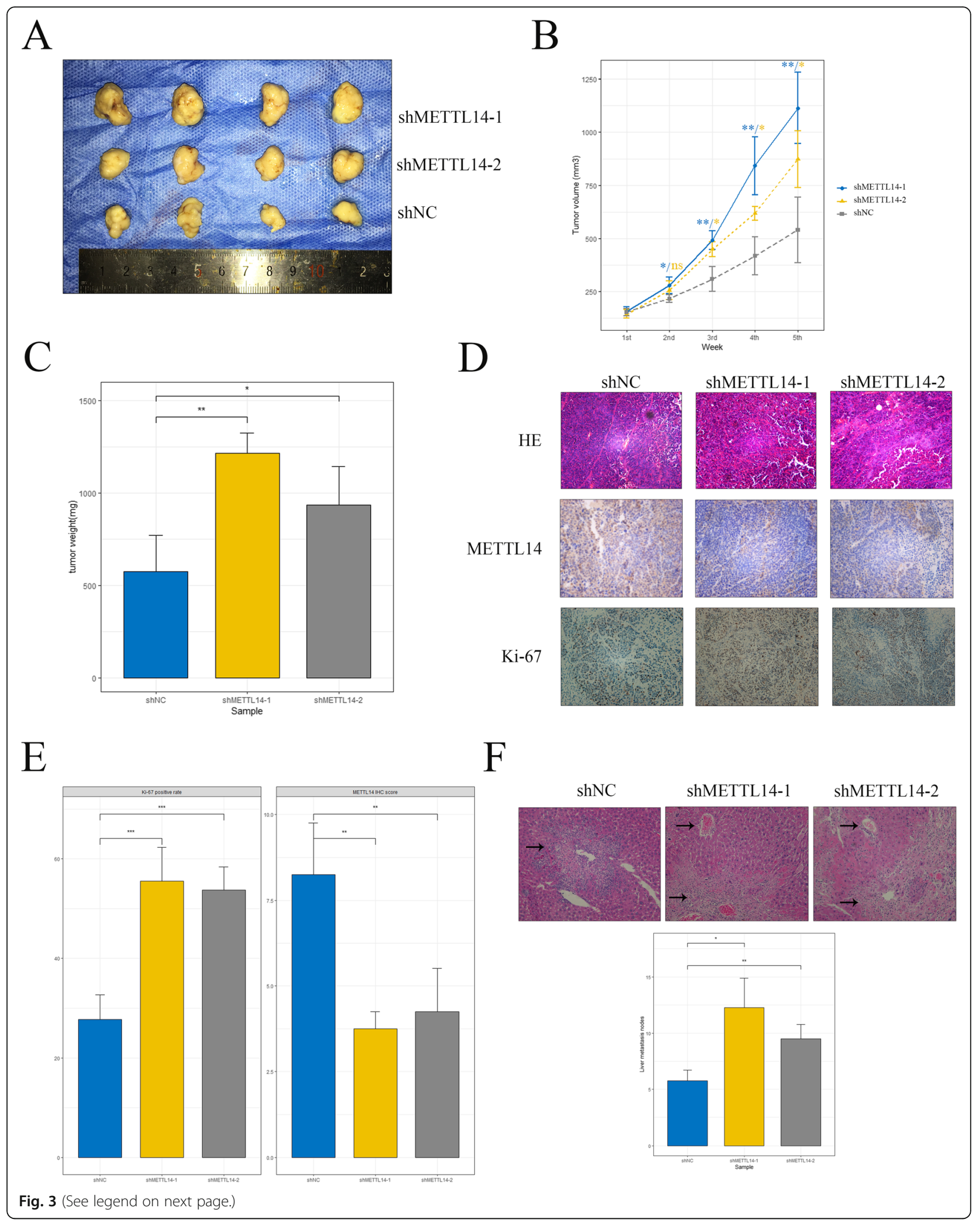


(See figure on previous page.)

Fig. 3 Knockdown of METTL14 promoted proliferation and invasion of CRC in vivo. a Xenograft tumors formed by HCT116-shMETTL14 or -shNC cells in nude mice. $\mathbf{b}-\mathbf{c}$ Quantitative analysis of tumor volume (b) and tumor weight (c) of xenografts. Tumor volume was compared at indicated time points and tumor weight was measured at the end point. ${ }^{*}, p<0.05,{ }^{*}, p<0.01$. $\mathbf{d}$ Xenograft tumor sections stained with hematoxylin and eosin (HE), METTL14 and Ki-67 were examined by immunohistochemistry. Magnification, 200x. e Analysis of METTL14 IHC score and Ki-67 positive rate in xenograft tumor tissue from different groups. ${ }^{*}, p<0.01$. $\mathbf{f}$ Analysis of metastatic liver nodules in different group of mice. Liver tissue of mice were counterstained with $\mathrm{HE}$ and typical metastatic lesions were indicated with black arrow. ${ }^{* *}, p<0.01 . P$ values were determined by Student's t-test

levels of methylated XIST (Fig. 5f). These results directly showed that METTL14 promotes m6A-methylation of $X I S T$, resulting in downregulation of this lncRNA and suppression of CRC proliferation and invasion. Intriguingly, we noticed that knockdown of WTAP almost totally abolished the m6A-methylation of XIST while METTL14 depletion significantly but only partly decreased m6A levels (Fig. 5f), which suggested that there might be other mechanisms involved.

m6A methylation is a marking procedure which needs to be recognized by $\mathrm{m} 6 \mathrm{~A}$ "reader" proteins for further disposition of target RNAs [24]. We next investigated the reader protein which recognized the m6A-methylation of $X I S T$ in order to mediate its downregulation. Analysis of previously reported iCLIP-sequencing data identified direct binding between XIST and the m6A readers YT521-B homology (YTH) domain family [20] which comprise three members of the YTHDF proteins (YTHDF1, YTHDF2, and YTHDF3), YTHDC1 and YTHDC2. To determine the binding between XIST and the potential reader proteins in CRC cells, we immunoprecipitated YTH family proteins with proper antibodies from the cell lysates and then measured the amount of bound XIST by quantitative PCR. As shown, YTHDF2 immunoprecipitants contained significantly higher level of XIST than control group (Fig. 6a and Additional file 10: Figure S8A). Consistently, RNA pull-down assay showed that YTHDF2, but not other readers, was significantly enriched by biotinlabeled XIST (Fig. 6b and Additional file 10: Figure S8B). Given that YTHDF2 was reported to facilitate m6Adependent RNA degradation under normal and stress conditions [25], we hence speculated that YTHDF2dependent m6A-RNA decay played an important role in the downregulation of XIST. As expected, knockdown of YTHDF2 resulted in remarkable augment of XIST expression in HCT116 and HT29 cells (Fig. 6c), which validated our hypothesis. Moreover, expression levels of YTHDF2 and XIST was negatively correlated with each other in TCGA dataset and our cohort (Fig. 6d). In addition, the decay rate of XIST was significantly slower in shYTHDF2 CRC cells, which directly showed YTHDF2 mediated the degradation of lncRNA XIST (Fig. 6e and Additional file 10: Figure S8C). Taken together, these findings suggested that METTL14-induced m6A process suppressed XIST expression through YTHDF2-dependent RNA degradation.

\section{Discussion}

N6-methyladenosine (m6A) of RNA has been proposed as a new layer of epigenetic regulation. This biochemical process is proved to play important roles in regulation of cell growth, differentiation, and self-renewal through controlling RNA splicing, translation and stability [23, 26, 27]. Previous studies identified $\mathrm{m} 6 \mathrm{~A}$ as a predominant modification of mRNAs [7, 23]. Strikingly, recent studies discovered that m6A was also present in the modification of ncRNAs. Among them, lncRNA XIST is highly methylated at special sites and $\mathrm{m} 6 \mathrm{~A}$ modifications were indispensable for XIST-mediated gene silencing. Accordingly, knockdown of methyltransferase like 3 (METTL3), or other accessory components including WTAP, RMB15 and RMB15B, significantly impaired XIST-mediated gene silencing [20].

Given the fact that IncRNA XIST promotes growth and invasion of colorectal cancer [19] and possesses abundant m6A sites (Additional file 7: Figure S5B and Additional file 1: Table S1) [20], it is reasonable for us to speculate that $\mathrm{m} 6 \mathrm{~A}$ may play an important role in regulating the biological behavior of CRC cells through inducing methylation of XIST. In the present study, we observed a decreased tendency of METTL14 from normal colon tissue to CRC (Fig. 1 ), suggesting the potential role of $\mathrm{m} 6 \mathrm{~A}$ in CRC tumorigenesis. Moreover, we demonstrated that METTL14 downregulation resulted in enhanced tumor growth and invasion of CRC both in vitro and in vivo (Fig. 2 and Fig. 3). In addition, METTL14 could serve as a prognostic factor for CRC patients (Fig. 1). Most importantly, we identified that lncRNA XIST was a direct downstream target of METTL14-mediated m6A modification (Fig. 4 and Fig. 5), unveiling the mechanisms by which METTL14 manipulated proliferative and invasive ability of CRC cells (Fig. 6f).

m6A process refers to a reversible chemical modification on RNA which is driven by a complex containing two catalytic components, METTL3 and METTL14. They form a heterodimer and catalyze the covalent transfer of methyl group to adenine with the assistance of WTAP, accomplishing the m6A installation process $[28,29]$. Previous studies suggested that dysregulation of these two enzymes contributed to human carcinogenesis. METTL3 was conspicuously upregulated in hepatocellular carcinoma (HCC) and consequently promoted proliferation and metastasis of $\mathrm{HCC}$ via silencing of tumor suppressor SOCS2 [11]. METTL3 was also elevated in 

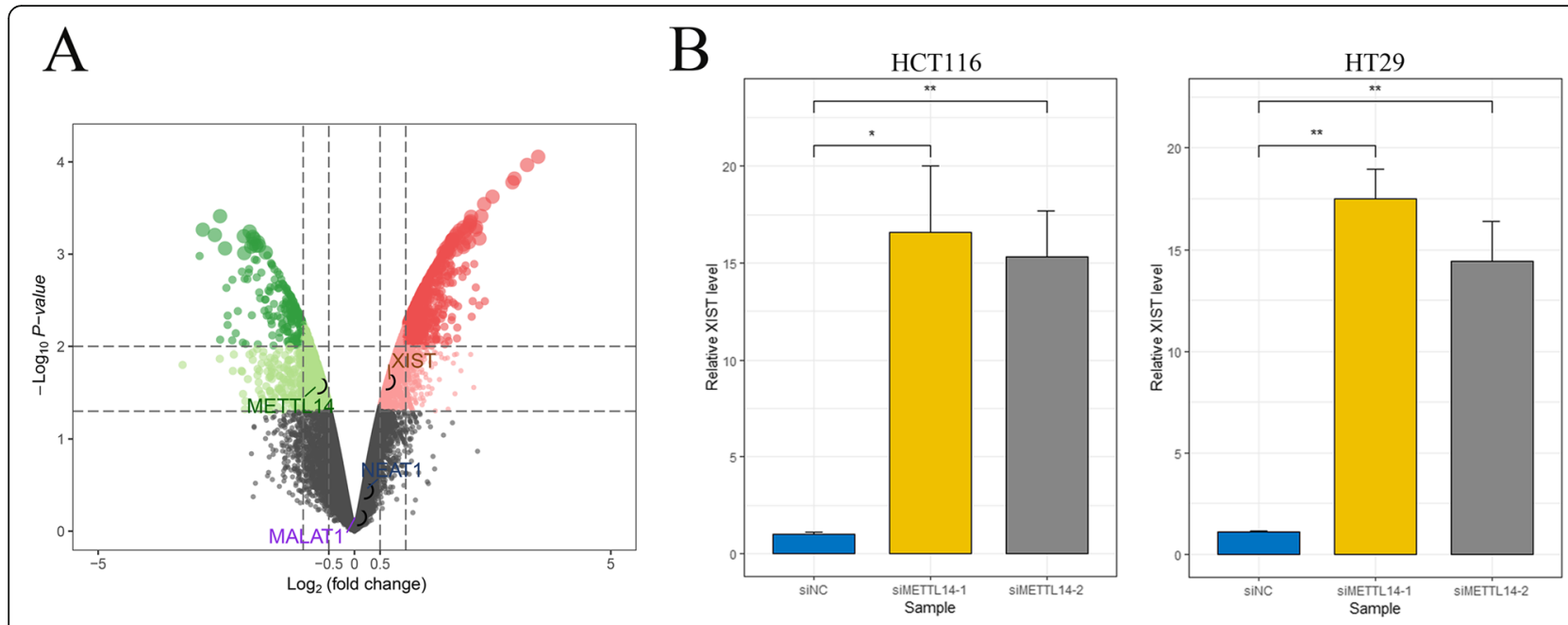

$\mathrm{C}$

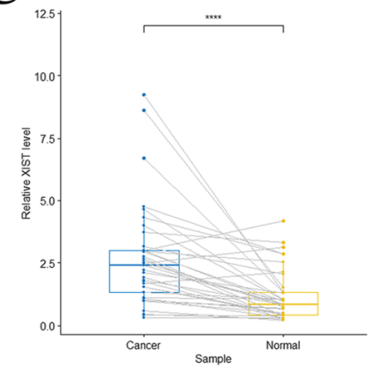

$\mathrm{F}$

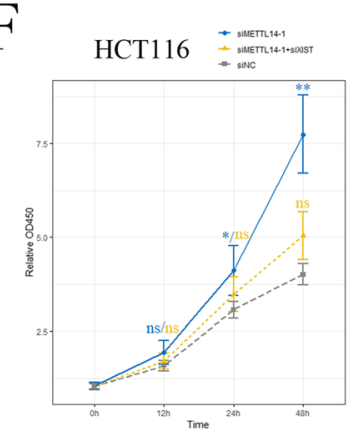

$\mathrm{D}$

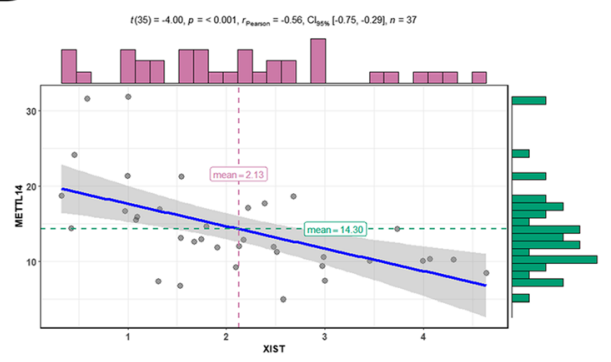

G

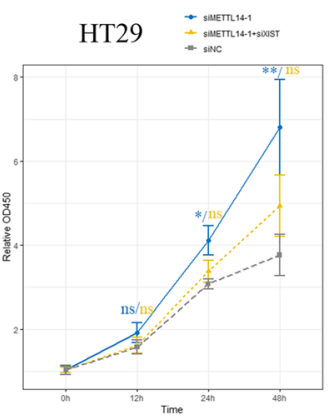

E
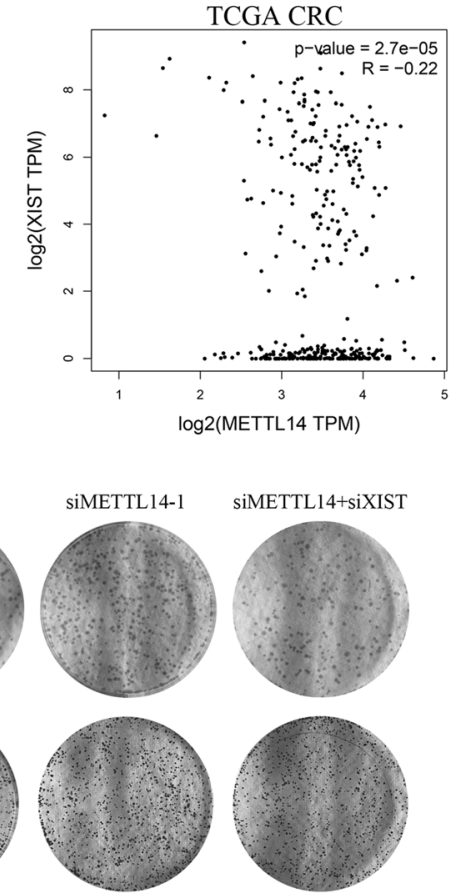

Fig. 4 METTL14 suppressed the growth and invasion of CRC by targeting IncRNA XIST. a Volcano plot showed DEGs between shMETTL14 and control cells. METTL14 and interested genes which were reported to possess multiple m6A residues were indicated with black circles. $\mathbf{b}$ The levels of XIST were examined by real-time PCR in HCT116 and HT29 cells transfected with siNC or siMETTL14, respectively. ${ }^{* *}, p<0.01$. c Real-time PCR showing expression levels of XIST in CRC tissue and paired normal tissue. ${ }^{* * *}, p<0.0001$. $\mathbf{d}$ and $\mathbf{e}$ Negative correlation of METTL14 and XIST expression in CRC tissue from our center (d) or from TCGA public dataset (e). R and $p$ values were as indicated. $\mathbf{f}$ and $\mathbf{g}$ Cell count assay and transwell assay showed the effects of METTL14 and XIST on the proliferative and invasive ability of CRC cells. Inhibition of XIST suppressed the increased cell growth $(\mathbf{f})$ and invasive ability $(\mathbf{g})$ resulted from knockdown of METTL $14 .{ }^{*}, p<0.05 .{ }^{* *}, p<0.01$. All data were representative of three independent experiments. Means \pm SD were shown. Statistical analysis was conducted using Student's $t$-test. Correlation of METTL14 and XIST waS evaluated by Pearson's correlation analysis

other solid tumors such as breast cancer [30], pancreatic cancer [31] and promotes growth, invasion and drug resistance of cancer cells. In contrast, METTL14 was down-regulated in HCC and resulted in decreased m6A level and enhanced the metastatic capacity of HCC cells [12]. In agreement with previous report, the present study also identified METTL14 as a tumor suppressor gene in CRC through down-regulating oncogenic lncRNA XIST, suggesting that m6A also represent a distinct form of epigenetic dysregulation in colorectal cancer. Moreover, although TCGA data showed that abnormal expression of METTL3 may exist in CRC, our 


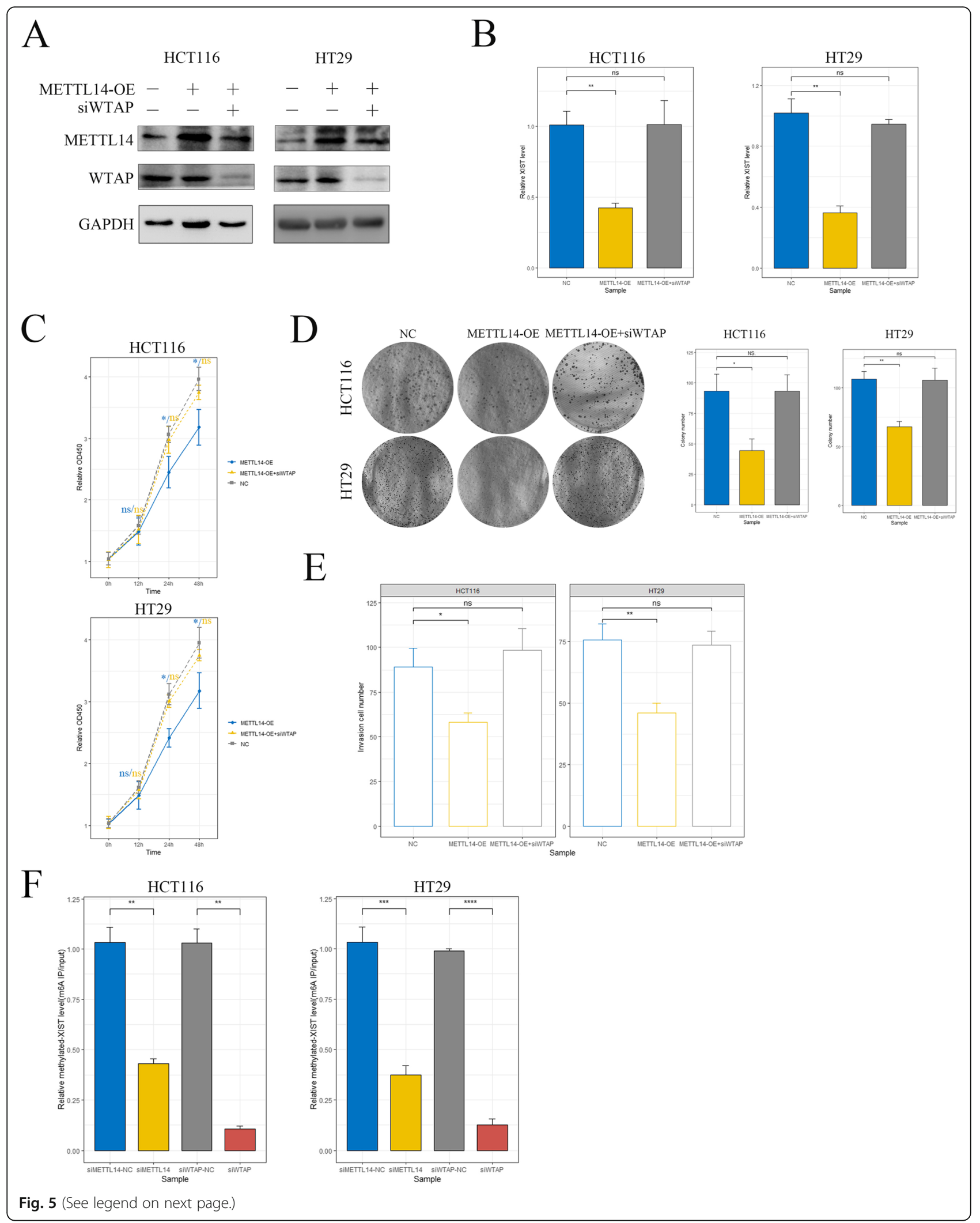


(See figure on previous page.)

Fig. 5 METTL14 down-regulated XIST through m6A methylation activity. a Two CRC cell lines were transfected with METTL14-overexpression (METTL14-OE) plasmids and siWTAP as indicated. Protein band intensity of METTL14 and WTAP were quantified by western blot assay and shown as fold change. Statistical histograms were not shown. b The alteration of XIST expression in METTL14-OE and METTL14-OE + siWTAP cells was analyzed by real-time PCR. ns, not significant, ${ }^{* *}, p<0.01$. $\mathbf{c}$-d The effects of WTAP and METTL14 on proliferative ability of CRC cells were measured by cell count assay (c) and colony formation assay (d). ns, not significant, ${ }^{*}, p<0.05,{ }^{* *}, p<0.01$. e Quantitative analysis of transwell invasion assay showing the effects of METTL14 and WTAP on invasive ability of CRC cells. ns, not significant, ${ }^{*}, p<0.05,{ }^{*}, p<0.01$. f Quantitative analysis of m6A-methylated XIST in control, siMETTL14-1 and siWTAP cells. Methylated XIST was immunoprecipitated with m6A antibody and then measured by real-time PCR. Equal amount of total RNA was used as input. ${ }^{* *}, p<0.01,{ }^{* * *}, p<0.001,{ }^{* * *}, p<0.0001$. All data were representative of three independent experiments. Means \pm SD were shown. $P$ values were determined by Student's $t$-test

knowledge about the link between METTL3 and CRC is still limited. A recent study reported that METTL3 could promote self-renewal of CRC cells through inducing m6A and subsequent upregulation of SOX2 [32]. Similarly, METTL3 and METTL14 also showed opposite effects in the regulation of $\mathrm{HCC}$ as above mentioned $[11,12]$. However, as METTL3 and METTL14 have to form a heterodimer to induce m6A methylation, it seemed to be contradictory for two components of a functional complex demonstrated definitely opposite effects on progression of cancer cells. We speculated that METTL3 and METTL14 might prefer different targets and lead to diverse downstream pathways. Moreover, the methyltransferase activating and determining the cell fate under certain conditions may have tissue and cellular specificity.

Whereas methyltransferase serve as the 'writer' of m6A, there are demethylases, FTO and alkB homolog 5 (ALKBH5), act as the 'eraser' to reverse the methylation. ALKBH5 catalyzes the direct removal of m6A [10], while FTO can sequentially oxidize m6A to N6hydroxymethyladeosine (hm6A) or N6-formyladenosine (f6A) [33], which are unstable and can be hydrolyzed to adenine. Notably, dysregulations of demethylases were also reported in cancers. FTO played an oncogenic role in acute myeloid leukemia through regulating target genes such as $A S B 2$ and $R A R A$ by reducing m6A levels in these mRNA transcripts [14]. ALKBH5 promoted cancer cell renewal and growth in breast cancer by removing m6A from NANOG mRNA, which in turn enhanced NANOG mRNA stability and pluripotency of cancer cells [34].

Similar to DNA methylation, the biological function of $\mathrm{m} 6 \mathrm{~A}$ is mediated through the selective recognition of $\mathrm{m} 6 \mathrm{~A}$ sites by the "reader" proteins [6, 24]. Among which, YTHDF2 is the first identified and the most extensively studied m6A reader. YTHDF2 can bind to m6A residues located in the untranslated region through $\mathrm{C}$-terminal YTD domain and render the targeted mRNA to processing bodies for subsequent degradation [35, 36]. Unlike YTHDF2, YTHDF1 promotes mRNA translation efficiency by interacting with EIF3 to promote rate-limiting step of translation for m6A-modified mRNAs [37]. YTHDC1 function as a regulator of RNA alternative splicing by recruiting serine and arginine-rich splicing factors (SRSFs) to its mRNAbinding regions near m6A sites [38]. Furthermore, heterogeneous nuclear ribonucleoprotein (hnRNP) could also serve as $\mathrm{m} 6 \mathrm{~A}$ reader to influence mRNA localization, alternative splicing and microRNA processing [39, 40]. Previous study reported that YTHDC1 recognized the METTL3-induced m6A methylation of XIST to mediate the transcriptional silencing of genes on the $\mathrm{X}$ chromosome [20]. However, here we found that m6A-methylated XIST was selectively recognized by YTHDF2 instead of YTHDC1, which was different from the results of previous study. We assumed that METTL14 might target different m6A sites of XIST comparing with METTL3, which led to diverse interactions with reader proteins and distinct downstream reactions. This question needs to be further clarified by single-nucleotide m6A detection methods.

Recent transcriptome-wide m6A profiling studies identified non-coding RNAs as both targets and regulators of m6A methyltransferases [23, 41, 42]. Subsequent studies established a model that METTL3 and METTL14 marked primary microRNAs for processing and maturation [12, 22 ]. Additionally, further investigations found that m6Amethylation was also presented in lncRNAs with m6A sites, such as XIST, NEAT1, [20], MALAT1 [40], and lincRNA1281 [43] and was responsible for the stability, structure, and function of these lncRNAs. Consistently, our proposition that m6A might target lncRNAs to affect cancer progression was validated in CRC cells. However, the above results indicated a complicated regulating network of m6A in different models of diseases. It is currently unclear what determines the target specificity and cellular heterogeneity of m6A modification in cancer cells. In addition, we wonder whether dysregulation of FTO or ALKBH5 exists in CRC and contributes to tumor formation or progression. Moreover, compared to recent advances in this aspect, the detailed m6A sites on XIST by which METTL14 targeted and regulated CRC cells was not precisely located in the present study. In addition, as most lncRNAs including XIST were produced in cell nuclei, the mechanisms mediating the export of XIST to cytoplasm for degradation was still not clear. Future investigations are necessary to answer these questions.

\section{Conclusion}

Collectively, we identified a 'METTL14-YTHDF2-IncRNA' regulating axis in CRC cells, which presented a systemic 


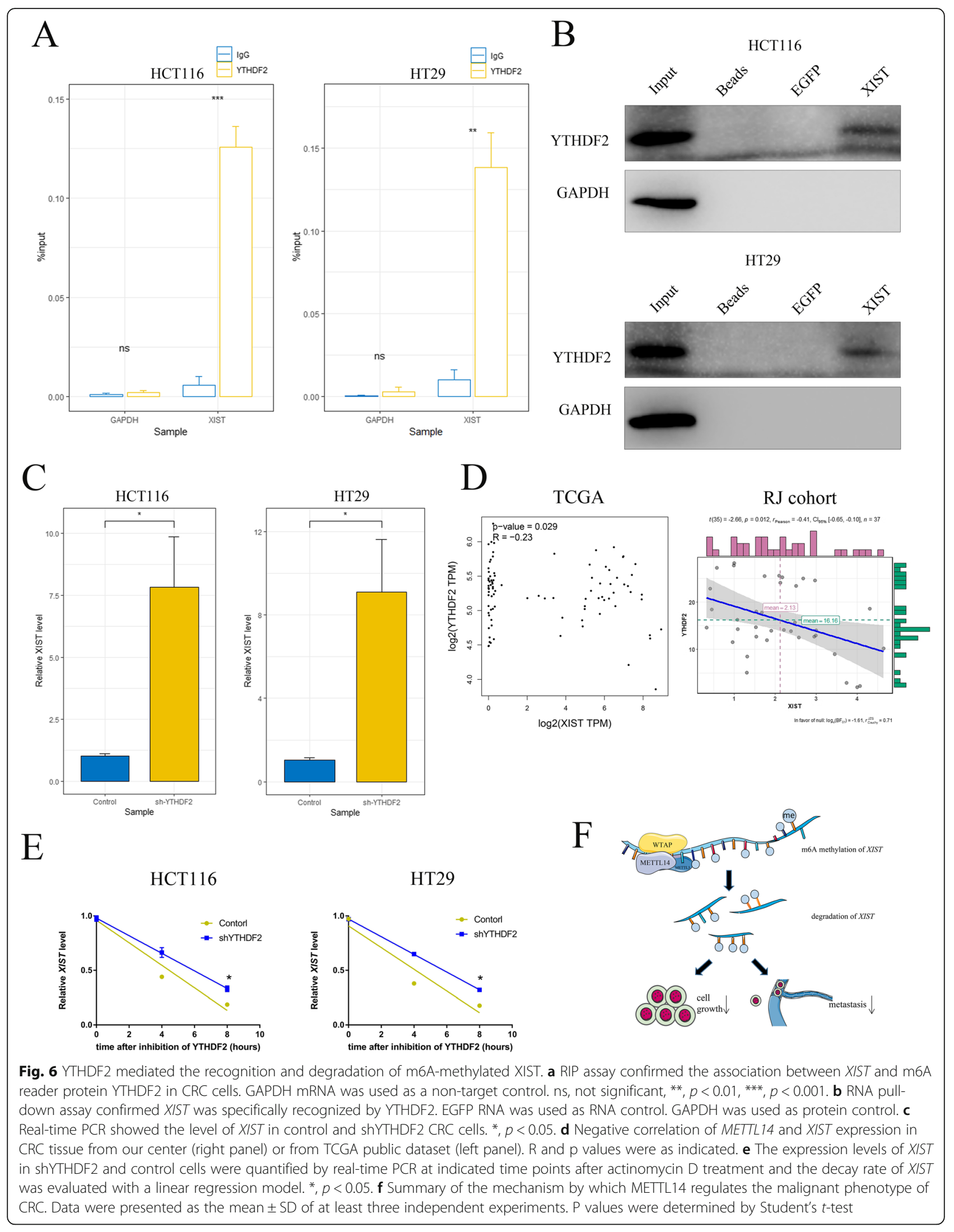


vision of the function and mechanism of $\mathrm{m} 6 \mathrm{~A}$ in $\mathrm{CRC}$ and revealed a novel dimension of cancer biology. Besides, by highlighting the crucial role and prognostic value of METTL14, our discovery may pave the way for developing new therapeutic strategies against CRC.

\section{Supplementary information}

Supplementary information accompanies this paper at https://doi.org/10. 1186/s12943-020-1146-4

Additional file 1: Table S1. m6A sites along XIST predicted by Me-DB online tools.

Additional file 2: Table S2. plasmids or siRNAs.

Additional file 3: Figure S1. (A-B) METTL14 was significantly downregulated $(A)$ while METTL3 was up-regulated (B) in CRC. RNA sequencing data was from TCGA database. $P$ values were as shown.

Additional file 4: Figure S2. (A) Comparison of predictive ability of METTL14 and TNM stage of RFS of CRC patients at 24 months. AUC values were as shown. (B) CRC patients from TCGA colon adenocarcinoma (COAD) and rectal adenocarcinoma (READ) dataset were classified into METTL14-high and -low group and difference between two groups was compared with Kaplan-Meier analysis. (C) Multivariate analysis of TCGA data showed that METTL14 was an independent risk factor for OS of CRC patients. (D) Quantitative analysis of western blots of Fig.2a. (E) Knockdown of METTL14 was performed with two shRNAs and was confirmed by western blots.

Additional file 5: Figure S3. The proliferative and invasive ability of two shMETTL14 cells were tested by cell count assay (A), colony formation assay (B) and transwell assay (C). ns, not significant, ${ }^{*}, p<0.05$, $* * * *, p<0.0001$.

Additional file 6: Figure S4. (A-C) METTL14 was positively correlated with regulation of non-coding RNA (ncRNA) metabolism (A), ncRNA processing (B), and RNA degradation (C). (D-F) METTL14 was negatively associated with growth factor binding (D), collagen trimer (E) and collagen binding (F). P values and normalized enrichment scores (NES) were as indicated.

Additional file 7: Figure S5. (A) Heatmap showed the DEGs between shMETTL14 and control cells. (B) Schematic diagram of m6A methylation sites of IncRNA XIST. Analysis was carried out by online tool MeT-DB (http://compgenomics.utsa.edu/methylation/). Potential m6A sites were shown as vertical bars. Three independent algorithms were applied. Details were shown in Additional file 1: Table S1. (C) Statistical histograms of Fig.4g. (D) Inhibition of XIST could attenuated the enhanced invasion of CRC cells resulted from METTL14 knockdown.

Additional file 8: Figure S6. Inhibition of XIST was performed in shMETTL14 cells and the proliferative and invasive ability were then tested by cell count assay (A), colony formation assay (B), transwell invasion assay $(C)$, respectively. The results showed that inhibition of XIST attenuated the increased cell growth and invasion of shMETTL14 cells.

Additional file 9: Figure S7. (A) Statistical analysis of protein bands from Fig.5a. (B) Representative images of transwell invasion assay of CRC cells with different expression status of METTL14 and WTAP.

Additional file 10: Figure S8. RIP $(A)$ and RNA-pulldown (B) assay detected negligible binding between XIST and YTHDF1, YTHDF3, YTHDC1 or YTHDC2. ns, not significant. (C) Knockdown of YTHDF2 was confirmed with western bolts.

\section{Abbreviations}

CRC: colorectal cancer; GSEA: Gene set enrichment analysis; IncRNA: long non-coding RNA; m6A: N6-methyladenosine; MeRIP: Methylated RNA immunoprecipitation; METTL14: methyltransferase-like14; RFS: recurrence free survival; WTAP: Wilms tumor-associated protein; XIST: $X$ inactivate-specific transcript; YTHDF2: YT521-B homology domain family 2

\section{Acknowledgements}

The authors would like to thank Dr. Chris Lou (Blogger, WeChat Official Accounts) for the bioinformatic technical support.

\section{Authors' contributions}

$X Y$ and JS designed the study. $X Y, S Z$ and $C Y H$ performed most of the experiment. PX, LYZ, ZRH and LZ performed the operation and followed-up the patients. $X Y$ and SZ analyzed data and drafted the manuscript. BF and $\mathrm{MHZ}$ provided the overall guidance. All authors read and approved the final Manuscript.

\section{Funding}

This work was supported by the National Natural Science Foundation of China (No.81802933 to Xiao Yang, No.81572818 to Minhua Zheng, No.81871984 to Jing Sun), the Guangci Distinguished Young Scholars Training Program (GCQN-2019-A07 to Xiao Yang) of RuiJin Hospital, Shanghai Jiao Tong University, Medical School.

\section{Availability of data and materials}

All data generated during this study are included either in article or in the additional files. RNA-seq data will be uploaded to GEO database in the future.

\section{Ethics approval}

All protocols for this study was reviewed and approved by Ruijin Hospital Institutional Ethics Committee.

\section{Consent for publication}

The content of this manuscript has not been previously published and is not under consideration for publication elsewhere.

\section{Competing interests}

The authors declare that they have no competing interests.

\section{Author details}

${ }^{1}$ Division of Gastrointestinal and Colorectal Surgery, Ruijin Hospital, Department of General Surgery, Shanghai Jiao Tong University, School of Medicine, Shanghai 200001, China. ${ }^{2}$ Shanghai Institute of Minimally Invasive Surgery, Ruijin Hospital, Shanghai Jiao Tong University, School of Medicine, Shanghai 200001, China.

Received: 22 September 2019 Accepted: 28 January 2020

Published online: 28 February 2020

\section{References}

1. Fitzmaurice C, Allen C, Barber RM, Barregard L, Bhutta ZA, Brenner $\mathrm{H}$, et al. Global, regional, and National Cancer Incidence, mortality, years of life lost, years lived with disability, and disability-adjusted life-years for 32 Cancer groups, 1990 to 2015: a systematic analysis for the global burden of disease study. JAMA Oncol. 2017;3:524.

2. Roth AD, Tejpar S, Delorenzi M, Yan P, Fiocca R, Klingbiel D, et al. Prognostic role of KRAS and BRAF in stage II and III resected Colon Cancer: results of the translational study on the PETACC-3, EORTC 40993, SAKK 60-00 trial. J Clin Oncol. 2010:28:466-74.

3. Kennedy RD, Bylesjo M, Kerr P, Davison T, Black JM, Kay EW, et al. Development and independent validation of a prognostic assay for stage II colon cancer using formalin-fixed paraffin-embedded tissue. J Clin Oncol. 2011:29:4620-6.

4. Suzuki MM, Bird A. DNA methylation landscapes: provocative insights from epigenomics. Nat Rev Genet. 2008;9:465-76.

5. Villanueva A, Portela A, Sayols S, Battiston C, Hoshida Y, Méndez-González J, et al. DNA methylation-based prognosis and epidrivers in hepatocellular carcinoma. Hepatology. 2015;61:1945

6. Fu Y, Dominissini D, Rechavi G, He C. Gene expression regulation mediated through reversible m6A RNA methylation. Nat Rev Genet. 2014;15:293.

7. Stower H. Transcriptome-wide N6-methyladenosine analysis. Nat Rev Genet. 2012;13:452

8. Schwartz S, Mumbach MR, Jovanovic M, Wang T, Maciag K, Bushkin GG, et al. Perturbation of m6A writers reveals two distinct classes of mRNA methylation at internal and 5' sites. Cell Rep. 2014;8:284. 
9. Lin S, Choe J, Du P, Triboulet R, Gregory RI. The m(6)a methyltransferase MEITL3 promotes translation in human Cancer cells. Mol Cell. 2016;62:335-45.

10. Zheng G, Dahl JA, Niu Y, Fedorcsak P, Huang CM, Li C, et al. ALKBH5 is a mammalian RNA Demethylase that impacts RNA metabolism and mouse fertility. Mol Cell. 2013;49:18-29.

11. Chen M, Wei L, Law CT, Tsang FH, Shen J, Cheng CL, et al. RNA N6methyladenosine methyltransferase METTL3 promotes liver cancer progression through YTHDF2 dependent post-transcriptional silencing of SOCS2. Hepatology. 2017;67:2254-70.

12. Ma JZ, Yang F, Zhou CC, Liu F, Yuan JH, Wang F, et al. METTL14 suppresses the metastatic potential of hepatocellular carcinoma by modulating N6methyladenosine-dependent primary MicroRNA processing. Hepatology. 2017;65:529.

13. Cui Q, Shi H, Ye P, Li L, Qu Q, Sun G, et al. M(6)a RNA methylation regulates the self-renewal and tumorigenesis of Glioblastoma stem cells. Cell Rep. 2017:18:2622-34.

14. Li Z, Weng H, Su R, Weng X, Zuo Z, Li C, et al. FTO Plays an Oncogenic Role in Acute Myeloid Leukemia as a N6-Methyladenosine RNA Demethylase. Cancer Cell. 2016;31:127.

15. Liu J, Ren D, Du Z, Wang H, Zhang H, Jin Y. m6A demethylase FTO facilitates tumor progression in lung squamous cell carcinoma by regulating MZF1 expression. Biochem Biophys Res Commun. 2018;502:456-64.

16. Jing F, Sun CC, Cheng G. Long non-coding RNA XIST acts as an oncogene in non-small cell lung cancer by epigenetically repressing KLF2 expression. Biochem Biophysical Res Commun. 2016;478:811-7.

17. Ren C, Li X, Wang T, Wang G, Zhao C, Liang T, et al. Functions and mechanisms of long non-coding RNAs in ovarian Cancer. Int J Gynecol Cancer. 2015;25:566.

18. Zhuang LK, Yang YT, Ma X, Han B, Wang ZS, Zhao QY, et al. MicroRNA-92b promotes hepatocellular carcinoma progression by targeting Smad7 and is mediated by long non-coding RNA XIST. Cell Death Dis. 2016;7:e2203.

19. Chen D, Chen L, Lu Y, Zhang D, Zeng Z, Pan Z, et al. Long non-coding RNA XIST expedites metastasis and modulates epithelial|[ndash]|mesenchymal transition in colorectal cancer. Cell Death Dis. 2017:8:e3011.

20. Patil DP, Chen CK, Pickering BF, Chow A, Jackson C, Guttman M, et al. m(6)A RNA methylation promotes XIST-mediated transcriptional repression. Nature. 2016;537:369.

21. Yang $X$, Zhu F, Yu C, Lu J, Zhang L, Lv Y, et al. N-myc downstream-regulated gene 1 promotes oxaliplatin-triggered apoptosis in colorectal cancer cells via enhancing the ubiquitination of BCl-2. Oncotarget. 2017;8:47709-24.

22. Alarcón CR, Lee H, Goodarzi H, Halberg N, Tavazoie SF. N6-methyladenosine marks primary microRNAs for processing. Nature. 2015;519:482-5.

23. Dai $D$, Wang $H$, Zhu $L$, Jin $H$, Wang $X$. N6-methyladenosine links RNA metabolism to cancer progression. Cell Death Dis. 2018;9:124.

24. Allis CD, Jenuwein T. The molecular hallmarks of epigenetic control. Nat Rev Genet. 2016;17:487.

25. Zhou J, Ji W, Gao X, Zhang X, Qian SB. Dynamic m6A mRNA methylation directs translational control of heat shock response. Nature. 2015;526:591.

26. Geula S, Moshitchmoshkovitz S, Dominissini D, Mansour AA, Kol N, Salmondivon M, et al. Stem cells. m6A mRNA methylation facilitates resolution of naive pluripotency toward differentiation. Science. 2015;347:1002.

27. Du Y, Hou G, Zhang H, Dou J, He J, Guo Y, et al. SUMOylation of the m6ARNA methyltransferase METTL3 modulates its function. Nucleic Acids Res. 2018;46:5195-208.

28. Liu J, Yue Y, Han D, Wang X, Fu Y, Zhang L, et al. A METTL3-METTL14 complex mediates mammalian nuclear RNA N6-adenosine methylation. Nat Chem Biol. 2014;10:93.

29. Ping $X L$, Sun BF, Wang $L$, Xiao W, Yang $X$, Wang WJ, et al. Mammalian WTAP is a regulatory subunit of the RNA N6-methyladenosine methyltransferase. Cell Res. 2014;24:177.

30. Cai X, Wang X, Cao C, Gao Y, Zhang S, Yang Z, et al. HBXIP-elevated methyltransferase METTL3 promotes the progression of breast cancer via inhibiting tumor suppressor let-7g. Cancer Lett. 2017;415:11-9.

31. Taketo K, Konno M, Asai A, Koseki J, Toratani M, Satoh T, et al. The epitranscriptome m6A writer METTL3 promotes chemo- and radio radioresistance in pancreatic cancer cells. Int J Oncol. 2018;52(2):621-9.

32. Li T, Hu P-S, Zuo Z, Lin J-F, Li X, Wu Q-N, et al. METTL3 facilitates tumor progression via an m6A-IGF2BP2-dependent mechanism in colorectal carcinoma. Mol Cancer. 2019;18:112.

33. Zhao X, Yang Y, Sun BF, Shi Y, Yang X, Xiao W, et al. FTO-dependent demethylation of N6-methyladenosine regulates mRNA splicing and is required for adipogenesis. Cell Res. 2014;24:1403.
34. Zhang C, Samanta D, Lu H, Bullen JW, Zhang H, Chen I, et al. Hypoxia induces the breast cancer stem cell phenotype by HIF-dependent and ALKBH5-mediated m6A-demethylation of NANOG mRNA. Proc Natl Acad Sc U S A. 2016:113:E2047.

35. Wang X, Lu Z, Gomez A, Hon GC, Yue Y, Han D, et al. N6-methyladenosinedependent regulation of messenger RNA stability. Nature. 2014;505:117-20.

36. Wang H, Zuo H, Liu J, Wen F, Gao Y, Zhu X, et al. Loss of YTHDF2-mediated m6A-dependent mRNA clearance facilitates hematopoietic stem cell regeneration. Cell Res. 2018;28:1035-8.

37. Wang X, Zhao BS, Roundtree IA, Lu Z, Han D, Ma H, et al. N6methyladenosine modulates messenger RNA translation efficiency. Cell. 2015;161:1388-99.

38. Xiao W, Adhikari S, Dahal U, Chen YS, Hao YJ, Sun BF, et al. Nuclear m(6)a reader YTHDC1 regulates mRNA splicing. Mol Cell. 2016;61:507-19.

39. Dominissini D, Moshitch-Moshkovitz S, Schwartz S, Salmon-Divon M, Ungar L, Osenberg S, et al. Topology of the human and mouse m6A RNA methylomes revealed by m6A-seq. Nature. 2012;485:201.

40. Alarcón CR, Goodarzi H, Lee H, Liu X, Tavazoie S, Tavazoie SF. HNRNPA2B1 is a mediator of $\mathrm{m}(6) \mathrm{A}$-dependent nuclear RNA processing events. Cell. 2015; 162:1299-308.

41. Gu S, Sun D, Dai H, Zhang Z. N 6 -methyladenosine mediates the cellular proliferation and apoptosis via microRNAs in arsenite-transformed cells. Toxicol Lett. 2018;292:1.

42. Chen T, Hao YJ, Zhang Y, Li MM, Wang M, Han W, et al. m6A RNA methylation is regulated by MicroRNAs and promotes reprogramming to Pluripotency. Cell Stem Cell. 2015;16:289-301.

43. Yang D, Qiao J, Wang G, Lan Y, Li G, Guo X, et al. N6-Methyladenosine modification of lincRNA 1281 is critically required for mESC differentiation potential. Nucleic Acids Res. 2018;46:3906-20.

\section{Publisher's Note}

Springer Nature remains neutral with regard to jurisdictional claims in published maps and institutional affiliations.
Ready to submit your research? Choose BMC and benefit from:

- fast, convenient online submission

- thorough peer review by experienced researchers in your field

- rapid publication on acceptance

- support for research data, including large and complex data types

- gold Open Access which fosters wider collaboration and increased citations

- maximum visibility for your research: over $100 \mathrm{M}$ website views per year

At BMC, research is always in progress.

Learn more biomedcentral.com/submissions 